\title{
19. Using MOVE (2)
}

When copying data from a field of one size to one of a different size, it is important to be aware of how this is done - as you could end up losing data.

\section{Alphabetic and Alphanumeric fields}

Data is copied one character at a time working from the left of the field to the right: if there is insufficient space in the field to which you are copying - then the righthand characters are lost; if there is too much space the remainder of the field is filled with spaces.

\section{e.g. MOVE NAME1 TO NAME2}

where NAME1 has been defined as PIC X(5) and NAME2 as PIC X(3).

If NAME1 contains $|f| r|e| d||$ - then after the MOVE has taken place NAME2 will contain $|\mathrm{f}| \mathrm{r}|\mathrm{e}|$

\section{e.g. MOVE NAME1 TO NAME3}

where NAME3 has been defined as PIC X(7) - then after the MOVE

NAME3 will contain $|f| r|e| d||||$

\section{Numeric fields}

Data is copied one digit at a time moving from the right of the field: so if the receiving field (i.e. the one you are copying to) is too small then the leftmost digits are lost; if it is too large then the excess space is filled with zeroes.

\section{e.g. MOVE NUM1 TO NUM2}

where NUM1 has been defined as PIC 9(5), NUM2 has been defined as PIC 9(3). If NUM1 contains $10|1| 2|9| 0 \mid$ - then after the MOVE has taken place NUM2 will contain $|2| 9|0|$

e.g. MOVE NUM1 TO NUM3

where NUM3 has been defined as PIC 9(7) - then after the MOVE

NUM3 will contain $101010|1| 2|9| 0 \mid$

If a numeric field contains decimal fractions then the number will be aligned around the decimal point after a MOVE and the excess digits at each end will be lost if the receiving field is too small.

e.g. MOVE NUM1 TO NUM2

where NUM1 is defined as PIC 999V99 and NUM2 is defined as PIC 99V9.

If NUM1 contains $121311|3| 2 \mid$ (i.e. 231.32) - then after the MOVE has taken place

NUM2 will contain $1311|3|$ (i.e. 31.3 )

e.g. MOVE NUM1 TO NUM3

where NUM3 is defined as PIC 9(5)V9(3) - then after the MOVE

NUM3 will contain $10|0| 2|3| 1|3| 2|0|$ (i.e. 00231.320). 